\title{
Relationships between Quantitative Electroencephalographic Alterations and the Severity of Hepatitis C Based on Liver Biopsy in Interferon- $\alpha$ Treated Patients
}

\author{
Satoshi Kamei ${ }^{1}$, Akihiko Morita ${ }^{1}$, Naohide Tanaka ${ }^{2}$, Masato Matsuura ${ }^{3}$, \\ Mitsuhiko Moriyama ${ }^{2}$, Takuya Kojima ${ }^{3}$, Yasuyuki Arakawa ${ }^{3}$, Yoshihiro Matsukawa ${ }^{4}$, \\ Tomohiko Mizutani $^{1}$, Teiichiro Sakai ${ }^{3}$, Kentaro Oga ${ }^{5}$, Hitoshi Ohkubo ${ }^{6}$, Hiroshi Matsumura ${ }^{7}$ \\ and Kaname Hirayanagi ${ }^{8}$
}

\begin{abstract}
Objective We have observed alterations of quantitative (q)-EEG findings occurring in interferon (IFN)- $\alpha$ treated chronic hepatitis $\mathrm{C}(\mathrm{CH}-\mathrm{C})$ patients, and found patient's age to be one factor influencing such EEG alterations. In the present study we evaluated the correlation between q-EEG alterations during IFN- $\alpha$ treatment and the severity of hepatitis based on liver biopsies.

Methods A total of $102 \mathrm{CH}-\mathrm{C}$ patients underwent blind, prospective and serial q-EEG examinations. The IFN- $\alpha$ was administered under the same therapeutic regimen to all patients. Serial EEGs were obtained before, at 2 and 4 weeks, and at 2-3 days after the conclusion of treatment. The absolute powers of each frequency band in different periods were determined by q-EEG. Staging (of fibrosis) and grading (of inflammatory cell infiltration) were scaled according to Desmet's classification. We evaluated the relationship between q-EEG and scales of staging or grading.

Results Age distributions did not differ significantly among stages or grades. As the stage or grade increased, the alterations of EEG during IFN- $\alpha$ treatment became more pronounced, and significant (repeatedmeasures analysis of variances; both, $\mathrm{p}<0.0001$ ).

Conclusion Alterations of the EEG occurring during IFN- $\alpha$ treatment became pronounced with more severe pathological findings for $\mathrm{CH}-\mathrm{C}$. Alterations in the EEGs during IFN- $\alpha$ treatment should be carefully monitored in $\mathrm{CH}-\mathrm{C}$ patients with severe pathological findings.
\end{abstract}

Key words: interferon- $\alpha$, quantitative-EEG, chronic hepatitis C, staging, grading

(Inter Med 48: 975-980, 2009)

(DOI: 10.2169/internalmedicine.48.1902)

\section{Introduction}

Alterations of brain waves on electroencephalograms
(EEGs) during treatment with interferon (IFN)- $\alpha$ have been described previously in several case reports (1-3). We have confirmed a diffuse slowing based on an analysis of blind, prospective and serial quantitative-EEG (q-EEG) examina-

\footnotetext{
${ }^{1}$ Division of Neurology, Department of Medicine, Nihon University Itabashi Hospital, Tokyo, ${ }^{2}$ Division of Gastroenterology and Hepatology, Department of Medicine, Nihon University Itabashi Hospital, Tokyo, ${ }^{3}$ Department of Neuropsychiatry, Nihon University Itabashi Hospital, Tokyo, ${ }^{4}$ Division of Hematology and Rheumatology, Department of Medicine, Nihon University Itabashi Hospital, Tokyo, ${ }^{5}$ Department of Neuropsychiatry, Nihon University Surugadai Hospital, Tokyo, ${ }^{6}$ Department of Internal Medicine, Nihon University Surugadai Hospital, Tokyo, ${ }^{7}$ Department of Internal Medicine, Itabashi Medical Association Hospital, Tokyo and ${ }^{8}$ Department of Hygiene and Public Health, Nihon University of Physical Education, Tokyo

Received for publication November 28, 2008; Accepted for publication March 11, 2009
}

Correspondence to Dr. Satoshi Kamei, skamei@med.nihon-u.ac.jp 
Table 1. Mean Values and Standard Deviations of Age for Each Stage and Grade Based on Liver Biopsies in 102 Subjects

\begin{tabular}{cccc}
\hline Findings of liver biopsy & Number of patients & $\begin{array}{c}\text { Mean } \pm \text { standard deviation } \\
\text { of patients' age } \\
\text { (years old) }\end{array}$ & $\begin{array}{c}\text { Difference in mean values between } \\
\text { different stages or grades } \\
\text { (Mann-Whitney U test) }\end{array}$ \\
\hline Stage (intrahepatic fibrosis) & 49 & $48.4 \pm 6.1$ & NS \\
\hline Mild & 38 & $51.9 \pm 7.4$ & NS \\
Moderate & 15 & $46.9 \pm 8.9$ & \\
Severe & & & \\
\hline Grade (inflammatory cell infiltration) & 43 & $58.2 \pm 6.0$ & \\
\hline Minimal & 30 & $50.6 \pm 7.1$ & \\
Mild & 14 & $51.3 \pm 6.8$ & \\
Moderate & 15 & $47.3 \pm 8.8$ & \\
Severe & &
\end{tabular}

tions undertaken in many patients with IFN- $\alpha$ treated chronic hepatitis $\mathrm{C}(4)$. We speculated that such diffuse slowing on the EEGs could reflect a mild encephalopathy due to the IFN- $\alpha$. We recently reported that the alteration of the q-EEG could be estimated clinically by the change in score on Mini-Mental State Examinations (5). We have also reported that the age of the patients was one of the factors affecting such alterations on the q-EEG (6). However, no other such factors have been reported. The present study was the first to evaluate the relationship between the alterations in q-EEG findings that occur during IFN- $\alpha$ treatment and the severity of hepatitis as estimated according to scales of staging and grading based on liver biopsies.

\section{Methods}

\section{Patients}

A total of 168 serial patients with chronic hepatitis $\mathrm{C}$ patients underwent our blind, prospective and serial q-EEG examinations, during the period from August 1997 to May 2007. These patients were independently registered at three different hospitals, viz. Nihon University Itabashi Hospital, Nihon University Surugadai Hospital, and Itabashi Medical Association Hospital, during the above period. All patients were investigated and treated under the same clinical regimen and conditions, including diagnostic criteria, q-EEG examinations, and IFN- $\alpha$ treatment, as reported previously (4). The clinical diagnosis of chronic hepatitis $\mathrm{C}$ was confirmed by serological findings of serum antibody for hepatitis $\mathrm{C}$ virus, histopathological findings obtained by liver biopsy, detection of the viral genome sequence for hepatitis $\mathrm{C}$ virus by the reverse transcriptase-polymerase chain reaction (RTPCR), serum liver function tests, and the clinical course of the patients. Staging (of intrahepatic fibrosis) and grading (of inflammatory cell infiltration) of the chronic hepatitis based on liver biopsies was scored according to Desmet's classification (7). Based on grading of histopathological findings according to the Desmet's classification (7), the patients with liver cirrhosis (LC) were excluded from the pre- sent study. A total of 102 patients ranging in age from 40 to 59 years were included in this study. All patients were alert during IFN- $\alpha$ treatment as graded according to the Glasgow Coma Scale. The mean values and standard deviations of patient age at each stage and grade of chronic hepatitis are listed in Table 1. There were no significant differences in mean age among stages or grades (Mann-Whitney $U$ test). IFN- $\alpha$ was administered intramuscularly at a dose of $9 \times 10^{6}$ IU daily for the first 4 weeks and then administered 3 times/ week for the following 20 weeks, according to the same regimen of IFN- $\alpha$ treatment. Informed consent to perform the present study was obtained from all patients. The serological hepatic function parameters of the 102 patients improved during the IFN- $\alpha$ treatment. The means and standard deviations for the values of AST (GOT) (normal: 8-38 IU/L) were $126.2 \pm 75.1$ before the treatment, $50.7 \pm 23.6$ at 2 weeks of treatment, $43.2 \pm 24.1$ at 4 weeks of treatment, and $40.4 \pm$ 23.1 IU/L after the treatment. The values for ALT (GPT) (normal: 4-44 IU/L) were 164.1 \pm 93.2 before the treatment, $60.4 \pm 33.2$ at 2 weeks of treatment, $55.0 \pm 29.1$ at 4 weeks of treatment, and 52.4 $\pm 27.1 \mathrm{IU} / \mathrm{L}$ after the treatment. No patients exhibited elevation of serological hepatic function parameters during IFN- $\alpha$ treatment. There were also no patients with significant elevation of serum ammonia concentration during this treatment. All of the patients gave informed consent to participate in the present study according to a protocol approved by the Ethics Committee for Human Studies at Nihon University.

\section{Q-EEG analysis}

The EEG recordings and q-EEG analysis employed in the present study were as described previously (4). Briefly, serial EEGs were obtained before the IFN treatment, at 2 and 4 weeks of treatment and at 2-3 days after the conclusion of treatment. The serial EEGs at 2 and 4 weeks of treatment were obtained during the period from 1 to 6 hours after the injection of IFN- $\alpha$. The EEGs in each subject were recorded on a magnetic optical disk from 16 electrode locations according to the 10-20 international system using a digital EEG instrument (Neurofax EEG-4518, Nihon Kohden, To- 
kyo, Japan). The EEGs were referenced to the ipsilateral earlobes. Sixty seconds of q-EEG data were selected visually from each subject and digitized at $128 \mathrm{~Hz}$ with a time constant of 0.3 , employing a high frequency filter of $60 \mathrm{~Hz}$. Thirty epochs with a duration of 2 seconds each were collected from the subsequent resting period with eyes closed for analysis of the q-EEGs. The procedure used for analysis involved the application of fast Fourier transformation of the collected EEG signals by Rhythm, version 10.0 (Stellate Systems Inc, Montreal, Quebec, Canada). The frequency ranges were divided into 6 bands, as follows: delta (1.17$3.91 \mathrm{~Hz})$, theta $1(4.30-5.86 \mathrm{~Hz})$, theta $2(6.25-7.81 \mathrm{~Hz})$, alpha $1(8.20-10.16 \mathrm{~Hz})$, alpha $2(10.55-12.89 \mathrm{~Hz})$, and beta (13.28-30.86 Hz). The absolute powers of each frequency band were calculated at each electrode location in all of the subjects. Each power value was obtained by integrating the appropriate part of the spectrum. The present quantitative analysis was carried out blindly during routine EEG work involving many other disease states, including epilepsy, cerebrovascular disease, encephalitis, meningitis, metabolic encephalopathy, and brain tumor, as well as in normal controls. The only knowledge that the EEG analyst (S. Kamei) possessed regarding each patient was the latter's identification number, and he had no other information regarding any other information concerning any of the studied subjects such as their clinical diagnosis, date of treatment, or type of treatment.

\section{Statistical analysis}

In September 2007, a statistical analyst (K. Hirayanagi) at another independent institute collected the analyzed q-EEG data, the data on patient ages, and that on histopathological findings on liver biopsy based on the Desmet's classification (7) for the 102 patients. Using Desmet's classification (7), the stage of intrahepatic fibrosis in each sample was classified as mild, moderate, or severe. The grade of inflammatory cell infiltration in each sample was classified as follows: minimal, mild, moderate, or severe. The distributions of the power values at each frequency band for each electrode location were evaluated in terms of their skewness and kurtosis. Based on findings regarding skewness and kurtosis, repeated measure analysis of variances (rANOVAs) was applied to the alterations in power values as the main factor among 4 different periods: before the IFN- $\alpha$ treatment, at 2 and 4 weeks of treatment, and after the treatment, with the frequency bands, electrode locations, and staging and grading on the hepatitis classifications as co-factors. SPSS statistical software Version 12.0 (SPSS Inc., Chicago, IL) was employed for statistical analysis. Relationships between qEEG variables and stages or grades were evaluated by post hoc ANOVAs (Scheffe's test). The level of significance for this study was 0.05 .

\section{Results}

There were no patients with IFN- $\alpha$ induced irreversible encephalopathy in the present study. Stages of the chronic hepatitis based on liver biopsies in the 102 subjects were distributed over the range from mild to severe fibrosis. Similarly, grades were also distributed from minimal to severe inflammatory cell infiltration. The results of serial q-EEG studies at each selected frequency of EEG during the IFN- $\alpha$ treatment for each staging and grading scale (Figs. 1, 2) revealed that increased slow waves (delta, theta 1 and 2) and decreased alpha 2 and beta waves were evident during the IFN- $\alpha$ treatment at all stages and grades. These EEG alterations during IFN- $\alpha$ treatment in the present study confirmed our previously reported observations (4). Moreover, the alterations in power values during the IFN- $\alpha$ treatment became more pronounced as the stage or grade of hepatitis increased. Statistical results obtained by rANOVAs (Table 2) for the interactions between the q-EEG alterations during the IFN- $\alpha$ treatment and differences of staging scale or grading scale were significant (both, $\mathrm{p}<0.0001$ ). Results of post hoc ANOVAs results (Table 3) also indicated significant differences in the alterations of absolute power values during the IFN- $\alpha$ treatment for all comparisons with increasing staging or grading scale in the case of the delta, theta 1 , and beta waves with the exception of several comparisons involving differences of only one grade or stage. There were no significant differences in the alterations of power values during the IFN- $\alpha$ treatment in the case of the alpha 1 and total power values. We also examined the correlations at each electrode location between severity based on liver biopsy findings and alteration of qEEG during the administration of IFN- $\alpha$. These correlations were significant for all electrode locations (frontal pole location $\mathrm{p}=0.03$ and $\mathrm{p}=$ 0.004 for stage and grade, respectively; frontal location $\mathrm{p}<$ 0.0001 for both stage and grade; temporal location $\mathrm{p}<0.0001$ for both; central location $\mathrm{p}=0.005$ and $\mathrm{p}=0.002$; parietal location $\mathrm{p}=0.002$ and $\mathrm{p}=0.01$; occipital location $\mathrm{p}=0.005$ and $\mathrm{p}=0.01)$.

There were only two patients with mild pyrexia (37.3 and $37.4^{\circ} \mathrm{C}$ ) at the time of q-EEG examination after 2 weeks of IFN- $\alpha$ administration, and no patient with pyrexia at the time of examination at 4 weeks. The two patients with mild pyrexia had findings of mild severity on liver biopsy. No significant effects of pyrexia on q-EEG were found.

\section{Discussion}

Although numerous patients have undergone IFN- $\alpha$ treatment, detailed assessments of the adverse effects of IFN- $\alpha$ on central nervous system function have not yet been presented. Evaluations of alterations in brain function have been presented in only three previous reports based on data from small numbers of patients who underwent EEG examinations (1-3). We recently confirmed a significant, diffuse slowing on q-EEGs that occurred in chronic hepatitis $\mathrm{C}$ patients during IFN- $\alpha$ treatment at a relatively low dosage (4). With such a low dosage of IFN- $\alpha$ administration to chronic hepatitis $\mathrm{C}$ patients, the diffuse slowing of the EEG is re- 

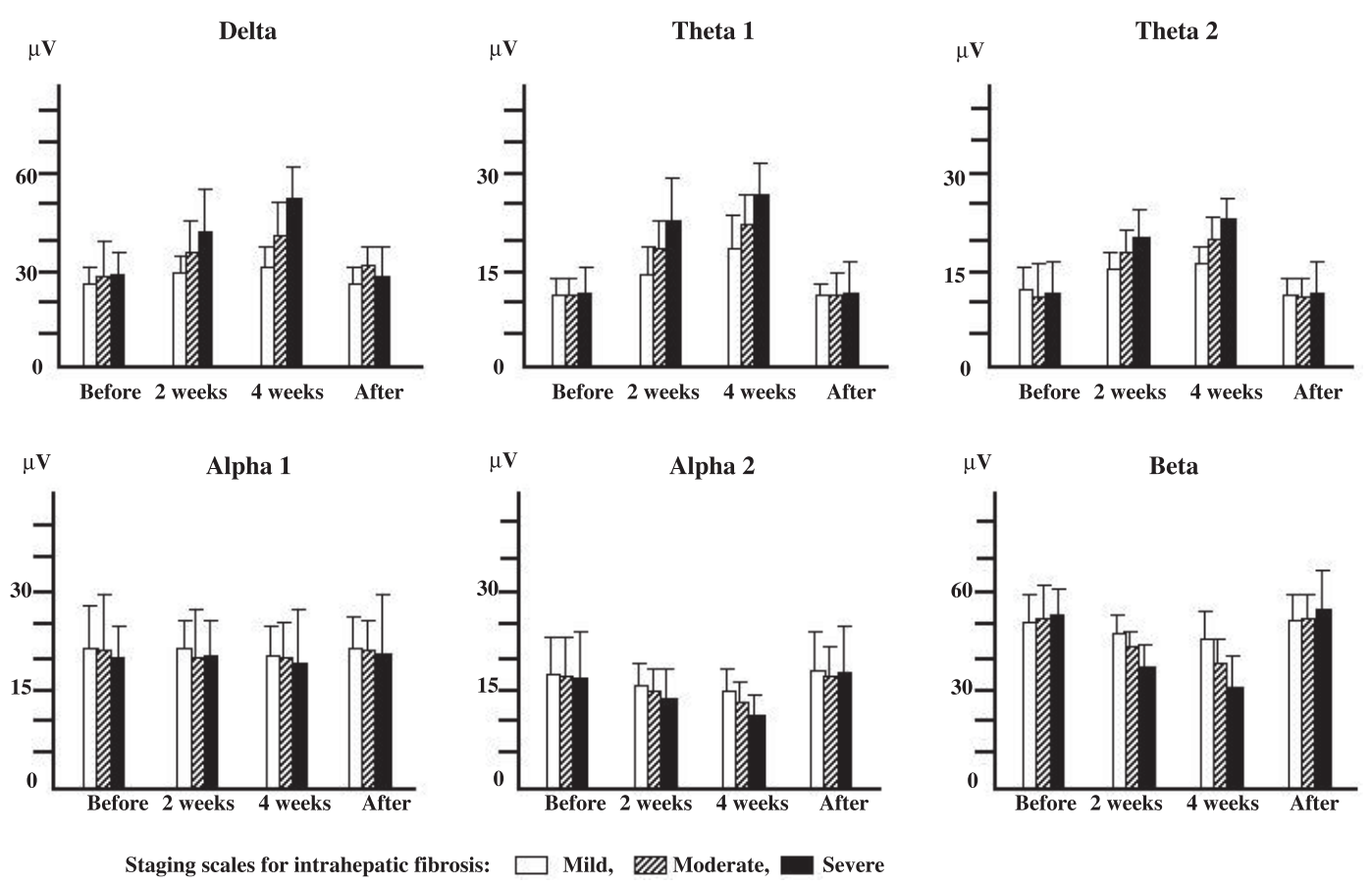

Figure 1. Alterations in absolute power values (means \pm standard deviations) by stage (of intrahepatic fibrosis) for each frequency band at the following 4 time points: before IFN- $\alpha$ treatment, at 2 and 4 weeks of treatment, and at 2-3 days after conclusion of treatment. Increasing power values for slow waves (delta, theta 1 and 2) and decreasing power values for alpha 2 and beta waves during IFN- $\alpha$ treatment, in comparison with those before and after IFN- $\alpha$ treatment, were evident for all stages. Moreover, alterations in power values became more pronounced as stage increased in all frequency bands except for alpha 1 and total power values.
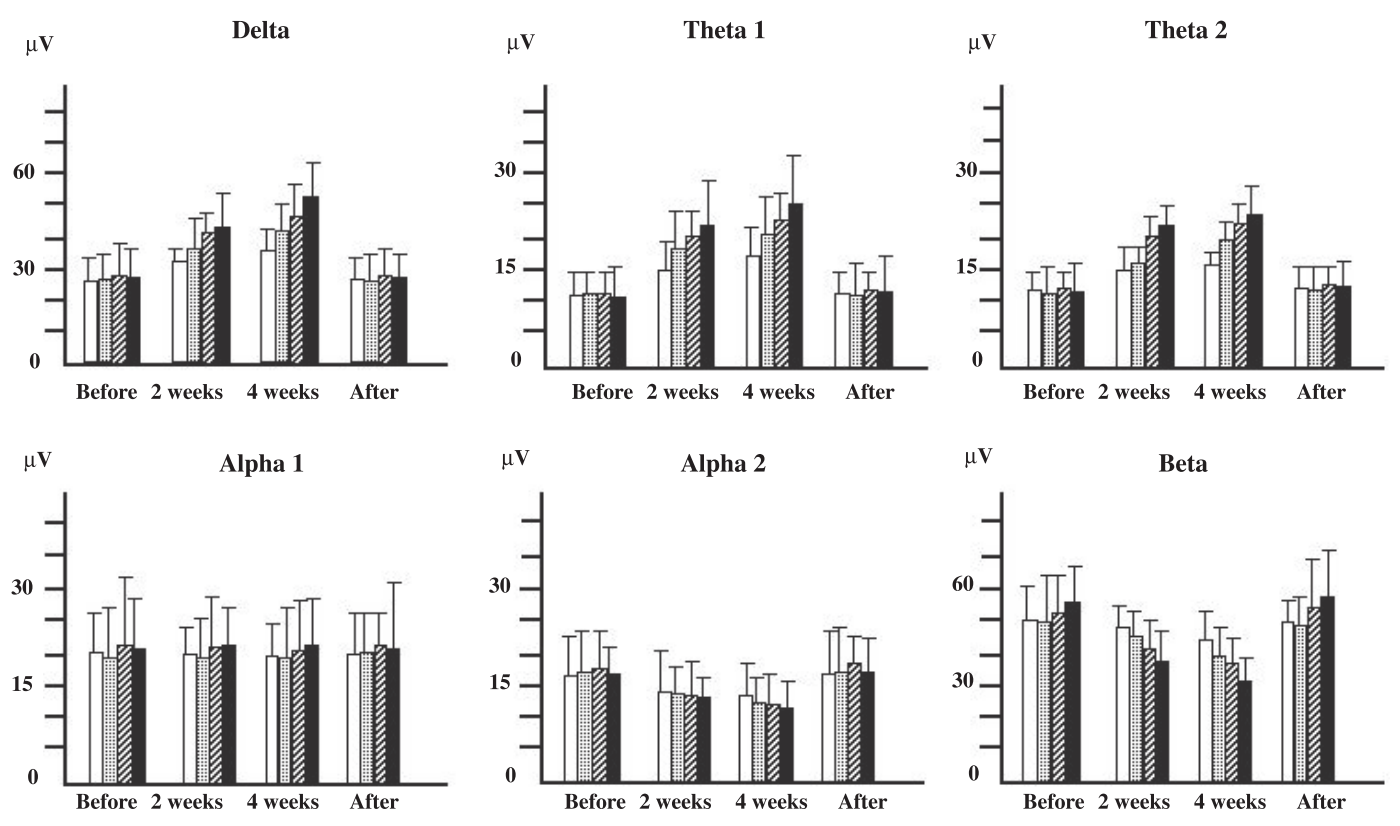

Grading scales for inflammatory cell infiltration: $\square$ minimal, 俩 mild, $\square$ moderate, $\square$ sever

Figure 2. Alterations in absolute power values (means \pm standard deviations) by grade (of inflammatory cell infiltration) for each frequency band at the following 4 time points: before IFN- $\alpha$ treatment, at 2 and 4 weeks of treatment, and at 2-3 days after conclusion of treatment. Increasing power values for slow waves (delta, theta 1 and 2) and decreasing power values for alpha 2 and beta waves during IFN- $\alpha$ treatment, in comparison with those before and after IFN- $\alpha$ treatment, were evident for all grades. Moreover, the alterations in power values became more pronounced with increase in grade in all frequency bands except for alpha 1 and total power values. 
Table 2. Repeated Measures Analysis of Variances between Alterations in Power Values during IFN- $\alpha$ Treatment and Stage (Intrahepatic Fibrosis) or Grade (Inflammatory Cell Infitraion)

\begin{tabular}{lc}
\hline \multicolumn{1}{c}{ Factors } & \\
\hline Alteration of power values during IFN- $\alpha$ treatment (Alteration of power values) & $\mathrm{p}<0.0001(\mathrm{~F}=30.338)$ \\
Alteration of power values $\times$ Difference of staging scale (Difference in stage) & $\mathrm{p}<0.0001(\mathrm{~F}=14.531)$ \\
Alteration of power values $\times$ Difference of grading scale (Difference in grade) & $\mathrm{p}<0.0001(\mathrm{~F}=12.071)$ \\
Alteration of power values $\times$ Frequency bands & $\mathrm{p}<0.0001(\mathrm{~F}=48.781)$ \\
Alteration of power values $\times$ Electrode location & $\mathrm{NS}$ \\
Alteration of power values $\times$ Difference in stage $\times$ Frequency bands $\times$ Electrode location & $\mathrm{NS}$ \\
Alteration of power values $\times$ Difference in grade $\times$ Frequency bands $\times$ Electrode location & $\mathrm{NS}$ \\
\hline
\end{tabular}

NS = not significant $\times$ = interaction.

Table 3. Statistical Comparisons by Post-hoc ANOVA of Alterations in Power Values during IFN$\alpha$ Treatment for Each Frequency Band between Stages and Grades

\begin{tabular}{|c|c|c|c|c|c|c|c|c|}
\hline \multirow{2}{*}{\multicolumn{2}{|c|}{$\begin{array}{c}\text { Comparison of stages and grades } \\
\text { based on biopsies }\end{array}$}} & \multicolumn{7}{|c|}{ Power values $(\mu \mathrm{V})$} \\
\hline & & \multirow{2}{*}{$\begin{array}{c}\text { delta } \\
* *\end{array}$} & \multirow{2}{*}{$\begin{array}{c}\text { theta } 1 \\
* * *\end{array}$} & \multirow{2}{*}{$\frac{\text { theta } 2}{*}$} & \multirow{2}{*}{$\begin{array}{c}\text { alpha } 1 \\
\text { NS }\end{array}$} & \multirow{2}{*}{$\begin{array}{c}\text { alpha } 2 \\
\text { NS }\end{array}$} & \multirow{2}{*}{ beta } & \multirow{2}{*}{$\begin{array}{l}\text { total } \\
\text { NS }\end{array}$} \\
\hline Stage & mild vs. moderate & & & & & & & \\
\hline (intrahepatic & moderate vs. severe & $* *$ & $* *$ & $* *$ & NS & $*$ & $* *$ & NS \\
\hline fibrosis) & mild vs. severe & $* *$ & $* *$ & $* *$ & NS & $*$ & $* *$ & NS \\
\hline \multirow{6}{*}{$\begin{array}{c}\text { Grade } \\
\text { (inflammatory cell } \\
\text { infiltration) }\end{array}$} & minimal vs. mild & $* *$ & $* *$ & $* *$ & NS & NS & $*$ & NS \\
\hline & minimal vs. moderate & $* *$ & $* *$ & $* *$ & NS & $*$ & $* *$ & NS \\
\hline & minimal vs. severe & $* *$ & $* *$ & $* *$ & NS & $*$ & $* *$ & NS \\
\hline & mild vs. moderate & $* *$ & $* *$ & $* *$ & NS & NS & $*$ & NS \\
\hline & mild vs. severe & $* *$ & $* *$ & $* *$ & NS & $*$ & $* *$ & NS \\
\hline & moderate vs. severe & $* *$ & $* *$ & $* *$ & NS & $*$ & $* *$ & NS \\
\hline
\end{tabular}

versible after completion of the treatment (4). Moreover, neuropsychiatric complications have been described as difficult to evaluate following IFN- $\alpha$ treatment in patients with chronic viral hepatitis (8). In view of the considerable numbers of patients undergoing IFN- $\alpha$ treatment, detailed information on the factors affecting EEG alterations due to IFN$\alpha$ treatment seems vital for prediction of the appearance of such adverse effects on brain function following IFN- $\alpha$ treatment. Patient age was recently identified as one of the factors involved in such alterations of EEGs during IFN- $\alpha$ treatment (6). However, no other factors affecting alterations of the EEG during IFN- $\alpha$ treatment have been reported. The findings of the present study indicated that severity of hepatitis based on liver biopsies is one such factor. However, there are some statistical limitations to the present study. Since we evaluated alterations of q-EEG during four different periods (pre-treatment, at 2 and 4 weeks of treatment, and post-treatment), the scales of severity based on liver biopsy findings were handled as continuous variables in rANOVA of present study.

The etiology of this type of encephalopathy remains unclear regarding whether it involves direct or indirect toxic effects on the central nervous system. Several possible indirect mechanisms can be considered. IFN plays a role in the production of secondary cytokines such as interleukin-1 and tumor necrosis factor (9). Neuroendocrine hormone alterations may also be induced by IFN. IFN displays structural and functional similarities to neuroendocrine hormones such as $\mathrm{ACTH}(10,11)$, and increased cortisol levels have been observed during IFN treatment. Such metabolic vulnerability might result in the diffuse slowing of brain waves observed on the EEG. LC is the most severe stage in Desmet's classification. Some degree of asymptomatic hypofunction of the brain might thus be evident even in patients with $\mathrm{CH}$, as in patients with LC. Our finding of a significant correlation between alteration of qEEG during IFN administration and the severity of liver biopsy findings suggests that the EEG alterations observed in the present study might be detected the brain hypofunction of mild encephalopathy due to IFN in addition to the some degree of brain hypofunction in the patients with severe $\mathrm{CH}$.

The diffuse slowing of EEG observed in the present study was reversible after the completion of treatment (Figs. 1, 2). This alteration of EEG was thus considered to be an asymptomatic and mild type of encephalopathy. However, the alterations of the EEG occurring during IFN- $\alpha$ treatment were marked in older aged patients and also in those with a high stage and grade based on liver biopsy findings. These findings suggest that the administration of IFN- $\alpha$ should be discontinued in patients with neuropsychiatric complications such as depression in the presence of EEG slowing. Serial EEG monitoring thus appears to be of value in detecting alterations of brain function during IFN- $\alpha$ treatment in chronic viral hepatitis patients, and alterations on serial EEGs should be carefully monitored in older patients and in those with severe stage and grade on liver biopsies. 


\section{Acknowledgement}

The present study was performed as one of the works of the Research Group for Comprehensive Clinical Studies on the Diagnosis, Treatment and Prevention of Neurological Disorders, which was supported by a Research Grant (18A-9) for Nervous and Mental Disorders from the Ministry of Health, Labour and Welfare, Japan, and a Grant from the Ministry of Science and Culture for the Promotion of Research Foundation.

\section{References}

1. Rohatiner AZS, Prior PF, Burton AC, Smith AT, Balkwill FR, Lister TA. Central nervous system toxicity of interferon. Br J Cancer 47: 419-422, 1983.

2. Smedley H, Kartrak M, Sikora K, Wheeler T. Neurological effects of recombinant interferon. Br Med J 286: 262-264, 1983.

3. Meyers CA, Scheibel RS, Forman AD. Persistent neurotoxicity of systemically administered interferon-alpha. Neurology 41: 672676, 1991.

4. Kamei S, Tanaka N, Matsuura M, et al. Blinded, prospective, and serial evaluation by quantitative-EEG in interferon-alpha-treated hepatitis-C. Acta Neurol Scand 100: 25-33, 1999.

5. Kamei S, Sakai T, Matsuura M, et al. Alterations of quantitative EEG and Mini-Mental State Examination in interferon- $\alpha$-treated hepatitis C. Eur Neurol 48: 102-107, 2002.

6. Kamei S, Oga K, Matsuura $M$, et al. Correlation between quantitative-EEG alterations and age of IFN- $\alpha$ treated hepatitis $\mathrm{C}$ patients. J Clin Neurophysiol 22: 49-52, 2005.

7. Desmet VJ, Gerber M, Hoofnagle JH, Manns M, Scheuer PJ.
Classification of chronic hepatitis: diagnosis, grading and staging. Hepatology 19: 1513-1520, 1994.

8. Saracco G, Rizzetto M. Therapy of Chronic Viral Hepatitis. In: Oxford Textbook of Clinical Hepatology. 1. 2nd ed. Bircher J, Benhamou J-P, McIntyre N, et al, Eds. Oxford University Press, Oxford, 1999: 939-954.

9. Arenzana-Seiededos F, Virelizer JL. Interferons as macrophageactivating factors, II: enhanced secretion of interleukin-1 by lipopolysaccharide-stimulated human monocytes. Eur J Immunol 13: 437-440, 1983.

10. Blalock JE, Smith EM. Human leukocyte interferon: structural and biological relatedness to adrenocorticotropic hormone and endorphins. Proc Natl Acad Sci USA 77: 5972-5974, 1980.

11. Blalock JE, Stanton JD. Common pathway of interferon and hormonal action. Nature 283: 406-408, 1980.

(C) 2009 The Japanese Society of Internal Medicine http://www.naika.or.jp/imindex.html 